\title{
Macroscopic and Microscopic Descriptions of the Plastic Deformation of Fcc Metals over a Wide Range of Strain and Temperature
}

\author{
T. CsanÁdi ${ }^{a, *}$, N.Q. Chinh ${ }^{b}$, J. GubiczA ${ }^{a}$ And T.G. LAngdon ${ }^{b, c}$ \\ ${ }^{a}$ Department of Materials Physics, Eötvös University Budapest H-1117 Budapest, Pázmány P. sétány 1/A., Hungary, \\ ${ }^{b}$ Departments of Aerospace \& Mechanical Engineering and Materials Science, University of Southern California \\ Los Angeles, CA 90089-1453, USA, \\ ${ }^{c}$ Materials Research Group, School of Engineering Sciences, University of Southampton \\ Southampton SO17 1BJ, U.K.
}

\begin{abstract}
The plastic behavior of face-centered cubic metals was investigated over a wide range of strain and testing temperature. The experimental stress-strain data were described using both macroscopic and microscopic, well-established relationships. The characteristics of these descriptions are discussed and compared with each other. The analysis of the characteristics leads to a definition of the low and high temperature deformation regions, where the kinetics of both the dislocation-multiplication and the dislocation-annihilation (recovery) are different. For pure aluminum, it is shown that the boundary between these two regions occurs at a homologous temperature of the order of $\approx 0.5 T_{\mathrm{m}}$ where $T_{\mathrm{m}}$ is the absolute melting temperature. From this analysis, correlations are also drawn between the macroscopic parameters describing the stress-strain relationship and the fundamental characteristics of the microscopic processes both at room temperature and elevated temperatures.
\end{abstract}

PACS: 61.66.Bi, 83.50.Uv, 65.40.-b, 81.40.Jj, 61.72.Cc

\section{Introduction}

Recently bulk nanostructured materials were extensively investigated because of their special properties and applications. Severe plastic deformation (SPD) is an efficient way to create bulk nanomaterials. The most commonly applied techniques are equal-channel angular pressing (ECAP) [1,2] and high-pressure torsion (HPT) $[3,4]$. In order to apply these methods efficiently, it is useful to understand the underlying processes and to describe the plastic deformation over a wide range of strain. Numerous models have been developed for the description of the plastic flow on both macroscopic and microscopic scales.

In this paper, a recently proposed exponential-power law constitutive equation [5] was applied to describe the macroscopic plastic flow behavior of four face-center cubic metals (Al, Au, $\mathrm{Cu}$ and $\mathrm{Ni}$ ). This constitutive equation incorporates the main features of both the conventional Hollomon-type power law relationship [6] and the Voce-type exponential relationship [7]. On the microscopic scale, a dislocation-based theoretical model [8] is used to simulate the established major micro-mechanisms describing the evolution of mobile and forest dislocations during plastic flow. The present work can be regarded as a continuation of a recently published study [9] where the correlations between the characteristics of the macroscopic and microscopic descriptions have been reported. In the present paper, the investigations of the relationships are extended to cover a wide temperature range.

* corresponding author; e-mail: csanadi@metal.elte.hu

\section{Experimental materials and procedures}

Two series of experiments were carried out by tensile tests. In the first series, different, high purity polycrystalline face-centered cubic (fcc) metals (Al, Au, Cu and $\mathrm{Ni}$ ) were investigated at room temperature. The samples were annealed for $1 \mathrm{~h}$ at temperatures of 673 , 773,873 , and $973 \mathrm{~K}$, respectively. In the second series, high purity $(99.99 \%)$ polycrystalline aluminum was tested at different temperatures $(293,353,393,433,473$, $623,673$, and $738 \mathrm{~K})$. More details about the samples are reported in the previous papers [10-12].

In order to study the plastic deformation over a wide range of strain, some annealed samples were subjected to uniaxial tensile tests while others were processed by ECAP which made it possible to achieve very high strains corresponding to the saturation state of the ultrafine-grained microstructure. In the first experiment, the tensile test was peformed at room temperature and a constant initial strain-rate of $10^{-3} \mathrm{~s}^{-1}$. The values of the $0.2 \%$ proof stress after ECAP were taken from the previous papers for $\mathrm{Al}$ [5], $\mathrm{Au}$ [13], $\mathrm{Cu}$ [5], and $\mathrm{Ni}$ [14]. In the case of the second experiment series, the aluminum samples were tested at the initial strain rate of $10^{-2} \mathrm{~s}^{-1}$.

\section{Results and discussion}

\subsection{Relationships between the characteristics of the} macroscopic and microscopic descriptions

\subsubsection{Macroscopic description}

It has been shown recently $[5,10]$ that the macroscopic stress-strain $(\sigma-\varepsilon)$ behavior may be fitted by a constitutive relationship of the form 


$$
\sigma=\sigma_{0}+\sigma_{1}\left[1-\exp \left(-\left(\frac{\varepsilon}{\varepsilon_{\mathrm{c}}}\right)^{n}\right)\right],
$$

where $\sigma_{0}, \sigma_{1}, \varepsilon_{\mathrm{c}}$ and the exponent $n$ are fitting parameters and the strain $\varepsilon$ is taken as the absolute amount of strain relative to the initial annealed state. Physically, the first constant, $\sigma_{0}$, is the sum of the friction stress and the flow stress related to the initial dislocation density at the onset of plastic deformation which is then described by the three fitting parameters, $\sigma_{1}, \varepsilon_{\mathrm{c}}$ and $n$. It is important to emphasize, as noted earlier $[5,9,10]$, that Eq. (1) incorporates the well-known Hollomon [6] and Voce-type [7] equations. Taking $n=1$, Eq. (1) leads to the Voce-type exponential equation

$$
\frac{\sigma_{\text {sat }}-\sigma}{\sigma_{\text {sat }}-\sigma_{0}}=\exp \left(-\frac{\varepsilon}{\varepsilon_{\mathrm{c}}}\right),
$$

where the saturation stress, $\sigma_{\text {sat }}$ is given as

$$
\sigma_{\text {sat }}=\sigma_{0}+\sigma_{1} \text {. }
$$

Furthermore, expressing Eq. (1) as a Taylor expansion at small strains leads to

$$
\sigma=\sigma_{0}+K \varepsilon^{n},
$$

which is the conventional power-law Hollomon relationship where $K$ is a constant. The values of parameters $\sigma_{0}$, $\sigma_{1}, \varepsilon_{\mathrm{c}}$ and $n$ for some fcc metals deformed at room temperature were determined by fitting Eq. (1) to the experimental stress-strain data and listed in Table 1 of Ref. [9]. An example for fitting is shown in Fig. 3 of Ref. [10]. The values of the parameter $n$ are between 0.69 and 0.86 , which differ significantly from the value of 1 characterizing the Voce-equation.

\subsubsection{Microscopic description}

During plastic deformation, the evolution of the dislocation density is affected by the formation and annihilation processes. The dislocation reactions may result in their trapping and therefore two types of dislocation densities may be identified: $\rho_{\mathrm{m}}$ and $\rho_{\mathrm{f}}$ for mobile and forest dislocations, respectively. The average total dislocation density, $\rho$, is then the sum of these two component parts so that $\rho=\rho_{\mathrm{m}}+\rho_{\mathrm{f}}$. Earlier studies $[5,9,10]$ showed that the dislocation-based theoretical Kubin-Estrin (KE) model [8] can be effectively used to describe the development of these dislocation densities. In this model, the approach requires a numerical solution of the following equations for pure metals:

$$
\frac{\mathrm{d} \rho_{\mathrm{m}}}{\mathrm{d} \varepsilon}=C_{1}-C_{2} \rho_{\mathrm{m}}-C_{3} \rho_{\mathrm{f}}^{1 / 2}
$$

and

$$
\frac{\mathrm{d} \rho_{\mathrm{f}}}{\mathrm{d} \varepsilon}=C_{2} \rho_{\mathrm{m}}+C_{3} \rho_{\mathrm{f}}^{1 / 2}-C_{4} \rho_{\mathrm{f}},
$$

where the terms containing the parameters $C_{i}$ are related to the primary competing microscopic processes occurring during plastic deformation such as the multiplication of mobile dislocations $\left(C_{1}\right)$, their mutual trapping $\left(C_{2}\right)$, their immobilization through interactions with forest dislocations $\left(C_{3}\right)$ and the advent of dynamic recovery $\left(C_{4}\right)$.

The evolution of the dislocation density as a function of strain was determined from the stress-strain curve using the Taylor equation. Fitting Eqs. (5a) and (5b) to the experimental dislocation density versus strain data for the fcc metals deformed at room temperature, it was shown $[9,10]$ that the value of the term, $C_{3} \rho_{\mathrm{f}}^{1 / 2}$ is at least one order of magnitude smaller than any of the other terms in Eqs. (5a) or (5b) over the entire strain regime. This means that during the plastic deformation of fcc metals at room temperature the reduction in the mobile dislocation density can be essentially attributed to the trapping mechanism related to the interaction between mobile dislocations (characterized by the term $C_{2} \rho_{\mathrm{m}}$ ), rather than to the effect of forest dislocations. The results of the numerical calculations based on Eqs. (5a) and (5b) also show that the values of $C_{2}$ and $C_{4}$ are practically the same for all metals, indicating that the same mechanism controls the trapping of mobile dislocations and the annihilation of forest dislocations.

Considering that the term containing parameter $C_{3}$ can be neglected, and that there is equality between $C_{2}$ and $C_{4}\left(C_{2}=C_{4}\right)$, it was also shown that the applied theoretical model expressed by Eqs. (5a) and (5b) can be simplified to give an analytical relationship for the evolution of the total dislocation density, $\rho$, as a function of strain given by

$$
\rho(\varepsilon)=\frac{2 C_{1}}{C_{4}}-\left(\frac{2 C_{1}}{C_{4}}-\rho_{0}\right)\left(1+\frac{C_{4} \varepsilon}{2}\right) \exp \left(-C_{4} \varepsilon\right),
$$

where $\rho_{0}$ is the initial dislocation density at $\varepsilon=0$ $\left(\rho(0)=\rho_{0}\right)$.

\subsubsection{Relationships between the characteristics of the macroscopic and microscopic descriptions}

On the basis of Eq. (6), the flow stress increment $\sigma_{\mathrm{p}, \mathrm{cal}}$, produced by the plastic strain can be calculated using the well-known Taylor equation as

$$
\sigma_{\mathrm{p}, \mathrm{cal}}=\alpha \mu b\left(\sqrt{\rho}-\sqrt{\rho_{0}}\right),
$$

where $\alpha$ is a geometrical constant including the Taylor factor $(\alpha=0.7$ was selected for all fcc metals in these calculations), $\mu$ is the shear modulus and $b$ is the magnitude of the Burgers vector. It should be noted that Eq. (7) is expressing the offset of the plastic deformation in term of the Taylor form operating when $\rho \geq \rho_{0}$, which is equivalent to the description of Eq. (1) when $\sigma \geq \sigma_{0}$. Therefore, the quantity, $\sigma_{\text {p.cal }}$ in Eq. (7) corresponds to the term containing $\sigma_{1}$ in Eq. (1). Considering the flow stresses obtained at high strains $(\varepsilon \rightarrow \infty)$ and at $\varepsilon_{\mathrm{c}}$, it was shown that [9]:

$$
\sigma_{1}=\alpha \mu b\left(\sqrt{\frac{2 C_{1}}{C_{4}}}-\sqrt{\rho_{0}}\right)
$$

and

$$
\varepsilon_{\mathrm{c}} \cong \frac{1}{C_{4}},
$$

respectively.

Figures 1 and 2 show the relationship between the quantities $\frac{\sigma_{1}}{\alpha \mu b}$ and $\sqrt{\frac{2 C_{1}}{C_{4}}}-\sqrt{\rho_{0}}$ (Fig. 1) as well as between the quantities $\varepsilon_{\mathrm{c}}$ and $\frac{1}{C_{4}}$ (Fig. 2) for different fcc metals deformed at room temperature and for $\mathrm{Al}$ samples deformed at different temperatures. In all cases, the datum points are fitted well by straight lines which go through the origin. The slopes of $\approx 1$ in these figures 
unambiguously confirm the validity of Eqs. (8) and (9) which describe the relationships between the characteristics $\left(\sigma_{1}, \varepsilon_{\mathrm{c}}\right)$ of the macroscopic description and $\left(C_{1}, C_{4}\right)$ of the microscopic mechanisms. Equations (8) and (9) also demonstrate that the values of $C_{1}$ and $C_{4}$ can be estimated from $\sigma_{1}$ and $\varepsilon_{\mathrm{c}}$. It should be emphasized that these figures have also an additional significance that they are confirming the validity of the simplification of Eqs. (5a) and (5b), i.e. the validity of Eq. (6).

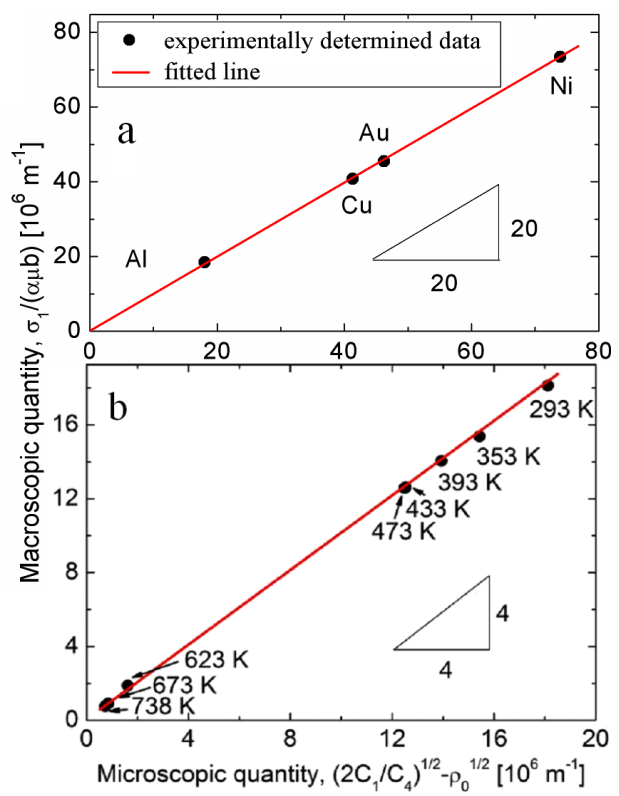

Fig. 1. The relationship between the characteristics of macroscopic and microscopic descriptions: $\frac{\sigma_{1}}{\alpha \mu b}$ versus $\left(\sqrt{\frac{2 C_{1}}{C_{4}}}-\sqrt{\rho_{0}}\right)$ for (a) different fcc metals deformed at room temperature [9] and (b) Al samples deformed at different temperatures.

\subsection{Low and high temperature regions of plastic deformation in $\mathrm{Al}$}

It was shown recently [11] that an increase in the testing temperature leads to the advent of the conventional secondary or steady-state stage of creep at relatively small strains. For the real work-hardening stage where $\partial \sigma / \partial \varepsilon>0$, the stress-strain relationship at high testing temperatures $\left(>0.5 T_{\mathrm{m}}\right)$ may be accurately described by Eq. (1) and the secondary creep behavior may be characterized unambiguously using a fitted saturation condition $\left(\sigma_{\text {sat }}\right)$ given by Eq. (3). Figure 3 shows the temperature dependence of the macroscopic parameter, $\sigma_{\mathrm{sat}}$ derived earlier for pure aluminum over a wide range of testing temperatures [11] where the data can be readily divided into two distinct regions corresponding to a low temperature behavior at $T<0.5 T_{\mathrm{m}}$ and a high temperature behavior at $T>0.5 T_{\mathrm{m}}$. This plot leads, therefore, to the conclusion that there are two separate and distinct temperature regimes occurring at low and high temperatures, respectively.

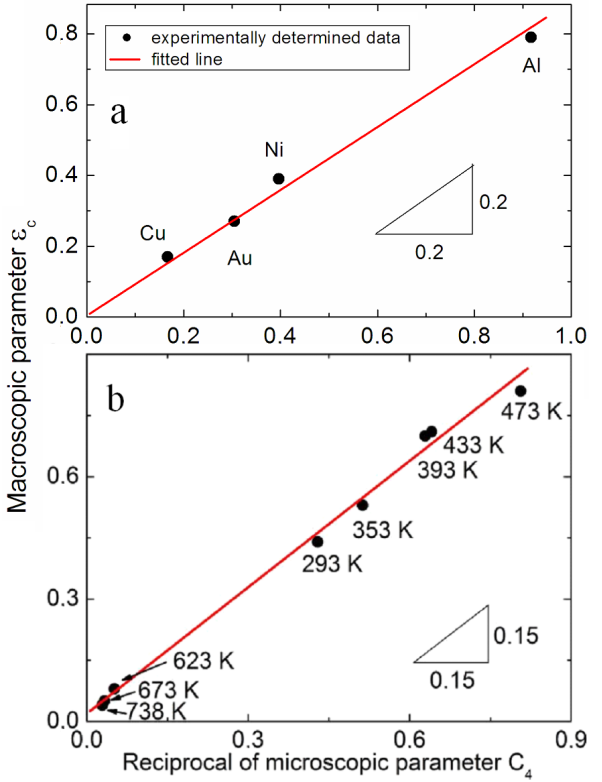

Fig. 2. The relationship between the characteristics of macroscopic and microscopic descriptions: $\varepsilon_{\mathrm{c}}$ versus $\frac{1}{C_{4}}$ for (a) different fcc metals deformed at room temperature [9] and (b) Al samples deformed at different temperatures.

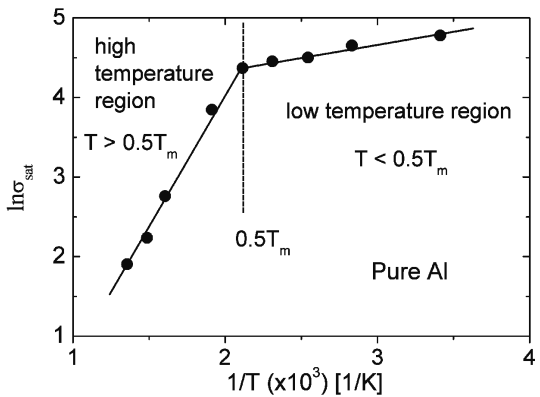

Fig. 3. The regions of low and high temperature flow in pure $\mathrm{Al}$ defined by the macroscopic saturation stress, $\sigma_{\text {sat }}$ given by Eq. (3) [11]. The unit of $\sigma_{\text {sat }}$ is MPa.

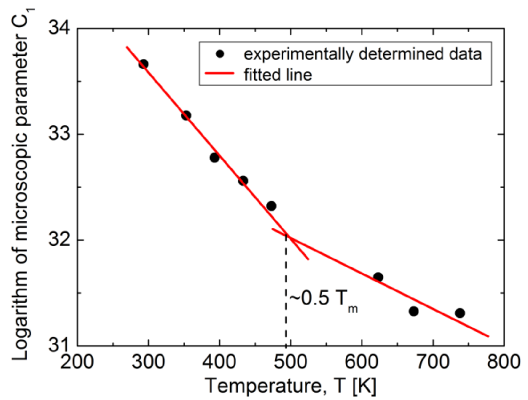

Fig. 4. The natural logarithm of the microscopic parameter $C_{1}$ for $\mathrm{Al}$ as a function of the deformation temperature. The unit of $C_{1}$ is $\mathrm{m}^{-2}$. 


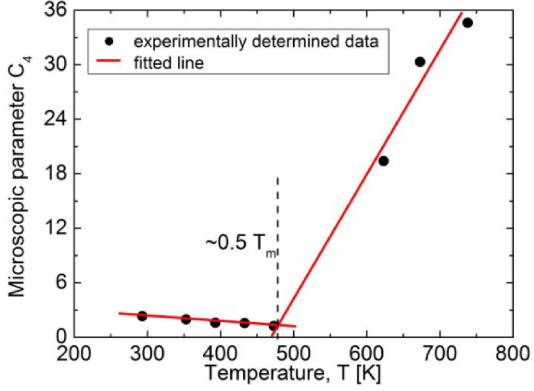

Fig. 5. The value of the microscopic parameter $C_{4}$ obtained for $\mathrm{Al}$ samples deformed at different testing temperatures.

Fitting Eq. (6) to the dislocation density versus strain data obtained experimentally from the stress-strain curve, the microscopic parameters $C_{1}$ and $C_{4}$ representing the dislocation multiplication and annihilation, respectively, were determined for pure $\mathrm{Al}$ over a wide range of testing temperatures. Figures 4 and 5 show the values of these microscopic parameters as a function of the temperature. It can be seen that in both cases the data, similar to the change of $\sigma_{\text {sat }}$ shown in Fig. 3, can be divided into low and high temperature regions. The results reveal two main points: (i) the transition at $0.5 T_{\mathrm{m}}$ between low and high temperature deformation regions can also be defined by the microscopic parameters $C_{1}$ and $C_{4}$, (ii) the changes of the dislocation multiplication $\left(C_{1}\right)$ and the dislocation annihilation $\left(C_{4}\right)$ parameters are stronger in the low $\left(T<0.5 T_{\mathrm{m}}\right)$ and the high $\left(T>0.5 T_{\mathrm{m}}\right)$ temperature regions, respectively. The break in the temperature dependences of parameters $C_{1}$ and $C_{4}$ indicates that the main processes in plastic deformation are controlled by various mechanisms with different activation energies below and above $0.5 T_{\mathrm{m}}$. For instance, the annihilation of dislocations at low temperatures may occur by cross-slip and/or pipe-diffusion controlled climb while the recovery at high temperatures is usually performed by lattice-diffusion controlled climb that has much larger activation energy than the low-temperature processes [15]. Further investigations are needed to study the relationships shown in Figs. 4 and 5 in more detail.

\section{Summary and conclusions}

1) The plastic behavior of fcc metals ( $\mathrm{Al}, \mathrm{Au}, \mathrm{Cu}, \mathrm{Ni}$ ) was investigated over a wide range of strain and testing temperature. The experimental stress-strain data were described by both macroscopic relationships and major microscopic mechanisms.

2) Quantitative correlations are presented to demonstrate the primary features of the microscopic processes determining the parameters describing the macroscopic evolution of the stress-strain relationships during plastic deformation, thereby confirming the physical meaning of the macroscopic characteristics that are often used as fitting parameters.

3) It was also found that the regions belonging to the high and low temperature deformations of $\mathrm{Al}$ can be distinguished by the changes of the microscopic parameters characterizing the multiplication of dislocations and the annihilation processes. The boundary between these two regimes occurs at a homologous temperature of $\approx 0.5 T_{\mathrm{m}}$ where $T_{\mathrm{m}}$ is the absolute melting temperature.

\section{Acknowledgments}

This work was supported in part by the Hungarian Scientific Research Funds, OTKA, grant No. K-81360 and K-67692 (CsT, NQC, JG), in part by the National Science Foundation of the United States under grant No. DMR-0855009 (TGL) and in part by the European Research Council under ERC grant agreement No. 267464-SPDMETALS (TGL). The European Union and the European Social Fund provided financial support to this project under grant agreement no. TÁMOP 4.2.1./B-09/ 1/KMR-2010-0003.

\section{References}

[1] R.Z. Valiev, R.K. Islamgaliev, I.V. Alexandrov, Prog. Mater. Sci. 45, 103 (2000).

[2] R.Z. Valiev, T.G. Langdon, Prog. Mater. Sci. 51, 881 (2006).

[3] A.P. Zhilyaev, T.G. Langdon, Prog. Mater. Sci. 53, 893 (2008).

[4] M. Kawasaki, B. Ahn, T.G. Langdon, Mater. Sci. Eng. A 527, 7008 (2010).

[5] N.Q. Chinh, Gy. Horváth, Z. Horita, T.G. Langdon, Acta Mater. 52, 3555 (2004).

[6] J.H. Hollomon, Trans. AIME 162, 268 (1945).

[7] E. Voce, J. Inst. Met. 74, 537 (1948).

[8] L.P. Kubin, Y. Estrin, Acta Mater. 38, 697 (1990).

[9] T. Csanádi, N.Q. Chinh, J. Gubicza, T.G. Langdon, Acta Mater. 59, 2385 (2011).

[10] N.Q. Chinh, T. Csanádi, J. Gubicza, T.G. Langdon, Acta Mater. 58, 5015 (2010).

[11] N.Q Chinh, J. Illy, Z. Horita, T.G. Langdon, Mater. Sci. Eng. A 410-411, 234 (2005).

[12] N.Q. Chinh, P. Szommer, T. Csanádi, T.G. Langdon, Mater. Sci. Eng. A 434, 326 (2006).

[13] T. Suzuki, A. Vinogradov, S. Hashimoto, Mater. Trans. 45, 2200 (2004).

[14] J. Gubicza, N.Q. Chinh, T. Csanádi, T.G. Langdon, T. Ungár, Mater. Sci. Eng. A 462, 86 (2007).

[15] H.J. Frost, M.F. Ashby, Deformation-Mechanism Maps: The Plasticity and Creep of Metals and Ceramics, Pergamon Press, Oxford, U.K. 1982. 